\title{
NOVOS ENCONTROS DE FLEBOTOMINEOS NO ESTADO DE SÃO PAULO, BRASIL, COM ESPECIAL REFERENCIA À LUTZOMYIA LONGIPALPIS
}

\author{
Oswaldo Paulo Forattini* \\ Ernesto Xavier Rabello * \\ Eunice Aparecida Bianchi Galati *
}

RSPU-B/306

\begin{abstract}
Forattini, O. P. et al. - Novos encontros de flebotomineos na Estado de São Paulo, Brasil, com especial referência à Lutzomyia longipalpis. Rcv. Saúde públ., S. Paulo, 10:125-8, 1976.
\end{abstract}

Resumo: Relata-se o encontro de várias espécies de Phlebotcminae, no Estado de São Paulo, Brasil, até agora raramente ou mesmo não assinaladas nessa região. São registrados novos achados de Lutzomyia longip:lpis e fornecidos alguns dados sobre as circunstancias desses encontros.

UNitermos: Phlebotominae. Lutzomyia longipalpis. Leishmaniose.

Os conhecimentos sobre a distribuição geográfica de Phlebotominae no Estado de São Paulo, Brasil, têm crescido de interesse, face a ocorrência de casos de leishmaniose tegumentar ter sido assinalada com certa freqüência nestes últimos anos. Assim sendo, os dados sobre esse assunto merecem ser divulgados com certa presteza, como foi o do encontro de Lutzomyia longipalpis, assinalado concretamente no município de Salto de Pirapora (Forattini et al., 1970).

No decurso de várias coletas realizadas no período de 1971 a 1975, foi possível observar a presença de espécies até agora raramente ou mesmo não registradas, no território paulista. Em grande número de casos, os exemplares foram surpreendidos abrigados em galinheiros experimentais utilizados para observações erológicas sobre populações naturais de triatomíneos.
Em outros, os espécimens foram obtidos através de capturas diretas com armadilhas tipo Shannon e tipo Disney (Forattini et al., 1976). Ėm número reduzido de casos o materidl foi constituído de insetos encontrados em domicílios ou peridomicílios. Os risultados obtidos foram os seguintes:

Lutzomyia corre $l$ limai Martins, Coutinho \& Luz, 1970.

15 espécimens ( 3 우 우 $12 \sigma^{x} \sigma^{x}$ ). Bairro do Pico, município de Taquarituba, em galinheiro experimental em outubro de 19'65.

Lutzomyia firmatoi (Barreto, Martins \& Pellegrin', 1956).

422 estécimens (332 우 $\left.90 \quad \sigma^{\pi} \sigma^{*}\right)$, assim distribuídos: Bairro da Carqueja, município de Cássia dos Coqueiros $(1) ?$

* Do Departamento de Epidemiologia da Faculdac de Saúde Pública da USP - Av. Dr. Arnaldo, 715 - São Paulo, SP — Brasil. 
FORATTINI, O. P. et al. - Novos encontros de flebotomíneos no Estado de São Paulo, Brasil, com especial referência à Lutzomyia longipalpis. Rev. Saúde públ., S. Paulo, 10; 125-8, 1976.

Em galinheiro experimental, em março de 1975.

Fazenda Jataí, municipio de Luís Antônio $\left(\begin{array}{lllll}77 & + & 67 & \sigma^{*} & \sigma^{*}\end{array}\right)$. Em armadilha tipo Disney, de julho de 1972 a outubro de 1973.

Sítio Samburá, municipio de Pedro de Toledo $(2$ 우 ). Em armadilha tipo Shannon, em novembro de 1973 (E. X. Rabello e O. P. Serra col.)

Bairro da Boiada, município de Cássia dos Coqueiros, (250 $\left.+9,170^{x} 0^{x}\right)$. Em galinheiros experimentais, de setembro de 1974 a dezem'. ro de 1975.

Bairro do Pico, município de Taquarituba $\left(2\right.$ 우, $\left.6 \sigma^{\star} \sigma^{\star}\right)$. Em galinheiro experimental, em outubro de 1975.

Lutzomyia lenti (Mangabeira, 1938). 98 espécimens $\left(\begin{array}{llll}46 & \text { 우 우 } 52 & \sigma^{x} & \sigma^{x}\end{array}\right)$, assim distribuídos:

Bairro Mil Alqueires, município de Pacaembu (1 $q)$. Em armadilha tipo Shannon, em ferereiro de 1971 (O. Mora col.).

Fazenda Brejāo, município de Guaira (45 우, $52 \sigma^{\pi}$ ). Em galinheiros experimentais, de setembro a novembro de 1975.

\section{Lutzomyia longipalpis (Lutz \& Neiva, 1912).}

584 espécimens (420 우,$\left.+ 164 \sigma^{\pi} \sigma^{\pi}\right)$ assim distribuídos:

Sítio do Sr. Armando Angelin, município de Pirapora do Bom Jesus (20 우 ㅇ, $\left.14 \delta^{x} \delta^{x}\right)$. Em isca humana, em maio de 1973 (N. C. B. von Atzingen col.).

Bairro da Carqueja, município de Cássia dos Coqueiros (399 우 우, $149 \sigma^{*} \sigma^{*}$ ).
Em galinheiros experimentais, de outubro de 1974 a dezembro de 1975.

Bairro da Boiada, municipio de Cássia dos Coqueiros (1 $\left.\sigma^{*}\right)$. Em galinheiro experimental, em dezembro de 1975.

Bairro Monte Alto, município de Cássia dos Coqueiros ( 1 q ). Em galinheiro experimental, em dezembro de 1975.

Lutzomyia longipennis (Barretto, 1946). 4 espécimens $\left(2\right.$ 우 우, $\left.2 \delta^{x} \sigma^{x}\right)$. Fazenda Jataí, município de Luís Antônio, em armadilha tipo Disney, de junho a setembro de 1972.

Psychodopygus guyanensis (Floch \& Abonnenc, 1945).

62 espécimens (14 우 오, $\left.180^{x} \sigma^{x}\right)$, assim distribuídos:

Guaratuba, municipio de: Santos (1 우). Em isca humana em março de 1972 (SLCEN' col.).

Porto Raso, Usina CBA, município de Miracatu (37 우, $\left.18 \sigma^{\pi}\right)$. Em armadilha tipo Shannon, em abril de 1972 (SUCEN col.).

Itamambuca, municipio de Ubatuba. Em isca humana, em julho de 1972. (SLCEN col.).

Bairro São Lourencinho, municipio de Pedro de Toledo $(4 \quad q$ q $)$. Em armadilha tipo Shannon, em abril de 1974. (O. P. Serra e J. M. S. Barata col.) .

Bairro São Lourencinho, município de Pedro de Toledo ( 1 ㅇ) . Em ambiente domiciliar, em abril de 1974. (O. P. Serra col.) 
FORATtiNi. O. P. et al. - Novos encontros de flebotomíneos no Estado de São Paulo, Brasil, com especial referência à Lutzomyia longipalpis. Rev. Saúde puibl., S. Paulo, 10: 125-8, 1976.

É de se ressaltar os novos encontros de Lutzomyia longipalpis que assim estende a outras localidades. a sua conhecida distribuição (Figura). Em se tratando de notório transmissor da leishmaniose visceral. não deixa de ser sugestivo o achado do díptero em ambientes constituílos por ecótopos artificiais. representados pelos galinheiros experimentais. Com efeito, esses abrigos. instalados em vários pontos do município de Cássia dos Coqueiros: mostraram-se particularmente atrativos para esse flebotomíneo. Note-se que sua presença somente foi assinalada naqueles abrigos colocarlos em campo aberto. fora das manchas residuais de florestas ali existentes. Taqueles instalados no Bairro da Carqueja. o resultado das coleta: levou à suposição de que o díptero tenha ali conseguido colonizar-se graças, talvez. à abundante matéria orgânica. representada pelas fezes de galinha. que assim serviria de alimento às formas larvais. De qualquer modo. a L. longipalpis é vetor de presença confirmada em território paulista. tendo assim sido obserrado procurando-os. ou mesmo colonizando-se em ecótopos artificiais. Acresce o fato de ter sido "surpreendido" sugando o homem. na captura levada a efeito no municipio de Pirapora do Bom Jesus. Tais informações serão certamente de utilidade para quem desejar pesquisar a eventual presença da endemia de calazar no Estado de Săo Paulo.

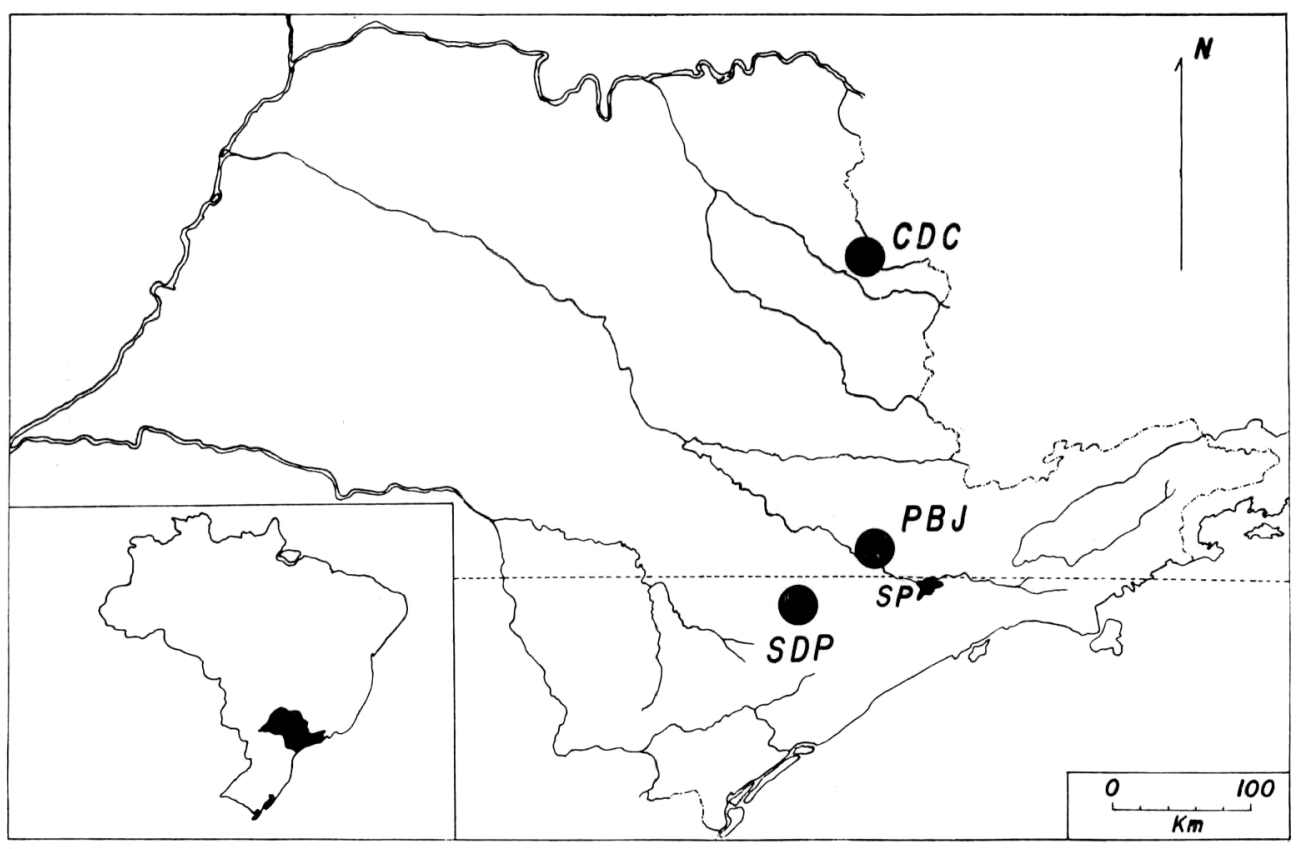

$$
\begin{aligned}
& \text { CDC - Cássia de Coqueiros } \\
& \text { PBJ - Pirapora do Bom Jesus } \\
& \text { SDP - Salto de Pirapora }
\end{aligned}
$$

Figura - Distribuicão atualmente conhecida de Lutzomyia longipalpis no Estado de São Paulo, Brasil. 
FORATTINi, O, P. et al. - Novos encontros de flebotomíneos no Estado de São Paulo, Brasil, com especial referência à Lutzomyia longipalpis. Rev. Salide päbl, S. Paulo, 10: $125-8,1976$.

RSPU-B/306

Forattini, O. P. et al. - [New finding of Phlebotominae in the State of S. Paulo, Brazil, with special refference to Lutzomyia longipalpis]). Rev. Saúde públ., S. Paulo, 10:125-8, 1976.

Summary: New records on Plltebotominae in the State of s. Panlo, Brazil, are reported. Special attention is dedicated to Lutzomyia longipalpis. Several behavioural aspects of these observations were recorded.

UNiterms: Phlebotominae. Lutzomyia longipalpis: Leishmaniasis.

REFERENCIA BIBLIOGRAFICA

FORATTINI, O. P. et al. - Sobre o encontro de Lutromyia longipalpis (Lutz \& Neiva, 1912) no Estado de São Paulo,
Brasil. Rev. Saíle píbl., S. Paulo, 4:99-100, 1970 .

Recebido para publicaço em 20/01/19\%6 A provado para publicasio em $05 / 01 / 19 \% 6$ 